\title{
An Efficient Routing Protocol for Video Stream in Clustered Wireless Sensor Networks
}

\author{
Sayyed Ahmad Hoseini ${ }^{1}$, Sayyed Majid Mazinani ${ }^{2}{ }^{*}$, Mehdi Shahedi ${ }^{3}$ \\ ${ }^{1}$ Computer Engineering Department, Payam Noor University, Mashhad, Iran. \\ 2 Electrical Engineering Department, Imam Reza International University, Mashhad, Iran. \\ ${ }^{3}$ Department of Computer, Science and Research branch, Islamic Azad University, South Khorasan, Birjand , \\ Iran. \\ *Corresponding author. Tel.: 00985137614550; email: smajid_mazinani@yahoo.com \\ Manuscript submitted July 26 2014; accepted September 30, 2014. \\ doi: 10.17706/ijcee.2014.v6.862
}

\begin{abstract}
In the time limit network traffic, especially video traffic, Packet delay is an important issue. However, there are several limitations in wireless sensor networks their main argument is that the network lifetime. The Congestion in Wireless Sensor Networks is significantly higher and thus severely affects the network performance leading to increased data loss and end to end delay and applications that require fast response time such as emergency preparedness and hostile environment surveillance pose challenging obstacles to wireless sensor network (WSN) protocols. A routing protocol must provide fast and reliable techniques for data propagation. In this study, we intend to approach in order to reduce energy consumption with maintaining the quality of service we provide to achieve improved network lifetime.
\end{abstract}

Key words: Congestion, energy efficiency, sink mobility, wireless sensor networks.

\section{Introduction}

In the proposed approach have been tried to improve the fallowing parameters:

1) The network life time

2) The end to end delay

A good way to routing that leads to cost decreasing is to use the communications between the cluster heads. In this way the hotspot problem is prevented [1].

In this Paper about the communication of mobile sink with the all network nodes. A way is proposed in wsn to optimizing apply .the new idea is to deliver data to mobile sink by forwarding from cluster heads (CHs).

As it is mentioned, one of the most important problems in forwarding delay sensitive data is end to end delay which occurs due to sending data to sink by some nodes [2]. Another problem is hotspot issue which indicates energy wasting in the sink surrounding nodes it causes the network life time comes shorter. The aims of our proposed approach are to create a permanently connection for transferring sensed delay sensitive data and to decrease energy wasting. The method we use is to divide the network nodes in small clusters, and then cluster heads are applied for sending out data to static and mobile sink.

We classify the sensed data to decrease energy consumption. Communication medium channels are also classified to route each packet in suitable path base on its importance. 
In the presented way, mobile sink stops near the cluster head and starts to move after a specified time duration .we utilize a priority queue in our scheme. The sink informs to cluster heads its movements towards them by sending a report packet. The packet is including of the waiting time duration and the time would be spent in the way to arrive the next cluster head. So if the information provided by the next cluster head doesn't have time limitless it can wait for arrival of the sink. The mentioned approach would increase the network lifetime and save the consumption energy. While a mobile sink arrives to a cluster head, a message include of the connected cluster head id to sink, stop duration of the mobile sink and the next cluster head id would be broadcasted into the network. This work decreases the traffic load on the static sink. In fact the data will be sent to static sink in times the mobile sink is moving and there is no connected cluster head to it.

\section{Related Works}

Submit the data gathering problem in WSNs is one of the field that is worked out so much and different methods is presented in this field.

In terms of energy consumption in different studies is mentioned two types of consuming energy models in the networks: energy consumption of the nodes in the WSN for the same total work (load) processed by the WSN. And the maximum energy dissipation per node. Emax is definitely one of many possible relevant indicators for the lifetime of the WSN. Ignoring the second energy consuming model has caused to disable a part of the network quickly [3], [4].

In [5] is proposed to deploy multiple static sinks. These static sinks make Partition the WSN into small Subsections that each of them has one static sink. This approach is similar to using a mobile sink for extending the lifetime of the nodes close to the sink but using several static sinks requires additional global communication for collecting all data at a single point. By simulation it was shown that the proposed scheme leads to energy efficiency and better data delivery ratio compared to schemes based on a single sink.

In static sink networks, the consumed energy average is low but this energy consumption increases in the nodes near the sink. In designing a "Robomote", which is an automatic platform (software and hardware) as a mobile node in a mobile sensor network to operate with a number of different applications. For example, it is possible to detect the network connection by having multiple nodes, the fluid moving in the desired location and network repair damaged and broken can be used. In addition, it can in order to distribute traffic load and energy consumption by the mobile sink can be used in different areas [6].

Increasing network lifetime, Mobile sink many initiatives to improve the lifetime of wireless sensor network is selected It is necessary to change the location or topology created by moving the sink over a network for the successful delivery of data to promote. As a result, the network lifetime achievements of sink mobility can be compensated by broadcasting high energy surplus is damaged. Due to this issue, Dual-Sink (double sink) were introduced. Both the mobile sink and static sink with dual sinks are used. Place the fluid sink only to a subset of nodes in the network each time it needs to be stopped. In another, Solution to the problem of deploying mobile data collection due to high traffic load and its bound around a sink due to static methods is proposed.

This is the method moving a sink that is used for this.

Protocol. MDC / PEQ proposed a mobile data collector (MDC) applies Mark Alert plays regularly. Sensor nodes receiving the warning will join the MDC cluster And Transmitted data to the data packets delay will update the MDC [6].

Warning signal to the power sensor nodes using a simple but effective way to run the configuration again .Mobility technique presented in the paper In fact, significantly reduce traffic And Thereby reducing the average number of mutations that data packets from sensor nodes to the sink or source that collects data 
transfer motion decreases.

Similar articles in this paper, which is the first mobile and fixed sink method used Except that sink in for a preset fixed station stops And begin to announce their presence to all the nodes within its range And They are also by given information send to the mobile sink instead of a static sink. Therefore, an optimization problem to choose the strategy that maximizes the traffic nodes to the minimum possible displacement would suggest. However, they are added in the total shortest path to make the best life for granted were an interesting research work is discussed in [7].

The authors in different researches have Presented Two approaches for mobile data collection. The first protocol is a simple passive method of collecting data, "wait until you hear the warning from a moving sink and then transfer." This technique significantly reduces the communication energy consumption of a mutation due to transfer data to a mobile sink running. However, according to the authors, such a solution for delay-sensitive applications due to the long period of a sensor node to a sink when moving between the transmissions ranges will wait. In the second approach, we utilize a static sink for the situations that mobile sink is absent in the interesting section .so in this state the data is sent to static sink instead of the mobile sink.

\section{Proposed Protocol}

Use In this paper, we focus on position of an abstract model with a circular shape and a mobile sink which moves along balanced circle with a fixed center. The mobile sink moves around the center of the circle .In this method, according to those already mentioned; we will notify the current location of the sink by the pull method.

In Our proposed approach, to prevent the energy loss in routing through clusters only cluster heads have the right to connect to a mobile sink. It is assumed, the cluster heads are located by user. On the other hand the sensors should be connected to the cluster heads among others which have higher quality of service in the network. Please see Fig. 1.
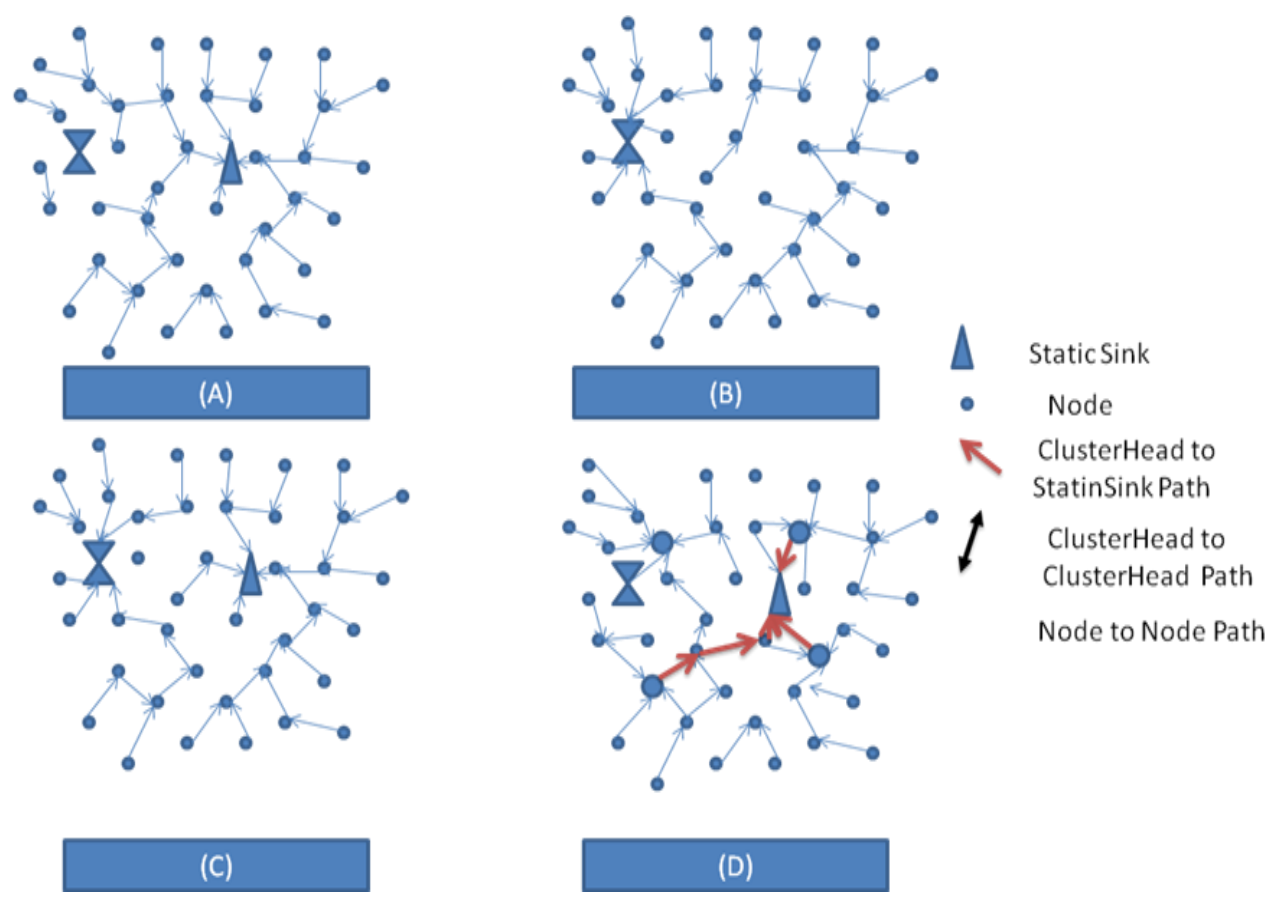

Fig. 1. (A) Mobile sink (B) Static sink (C) Dual sink (D) EEQR. 
In Static Solution (A) is a classic architecture of WSN includes randomly deployed sensor nodes near to which Base Station (BS) is placed .Sink is connected to internet through any other wired or wireless network.

Sink gives instructions to sensor nodes and gathers sensed data from them .As per the application requirement, sensor nodes, sense the desirable physical phenomenon and locally do the data aggregation to avoid communication of redundant data. It sends the sensed aggregated data to sink using hop-by-hop communication. Once a sink receives sensed data, it processes and forwards it, which is easily accessible to the user through internet. Nodes near to the sink deplete their battery power; problem is termed as hotspot problem. As a result, though the nodes farther from the sink have significant energy left but their energy cannot be utilized as the nodes near to the sink have depleted their energy. Hence, sensed data cannot be sent to the sink across hotspot or energy hole near the sink. It can significantly minimize network lifetime. Static nature of the sink is the main reason behind the hotspot problem, as all the time same nodes near the sink has to forward the data. Main advantage of the static sink approach is that it involves less end-to-end delay [5], [8].

In Mobile Solution (B) nodes near the mobile sink connect to Mobile Sink and Other Nodes also Connect to Connected Nodes to mobile sink but since the mobile sink always moves the paths connected to the mobile sink will be destroyed and a Rerouting should be performed to Find New Path to the Mobile Sink

In Dual Sink (C) If mobile sink Connect to a Node other nodes of neighbor of the node tries to Connect and Send Data from Mobile sink and Other Node Send Data to the Static Sink

In EEQR (D) Network is divided by Cluster heads and each part of network Connects to one Cluster Head and Sends Data to the Cluster head and if Mobile Sink is in the range of Cluster Head, the data from cluster head can be forwarded to the mobile sink. And another Cluster Heads Send Data to the static sink [2].

All The sensor nodes in the WSN will form group called clusters, and each cluster elects their $\mathrm{CH}$ in distributed manner using Local information. Each $\mathrm{CH}$ collects sensor data from other nodes in the cluster and transfers the aggregate data to the next Super node, which sends it to the static/mobile sink using multi-hop super-node communication. CH performs Extra duty since it collects, aggregates, and relays data from/to its member nodes. To achieve balanced energy consumption, the role and responsibility as a $\mathrm{CH}$ is rotated over the time among the member nodes in the cluster .In the proposed EEQR Protocol, the following assumptions are considered. WSN contains two types of nodes: normal node and few nodes equipped with extra energy resource, termed as super-Node. Super-nodes are less in number than normal nodes.

There is a static sink located at the center of the network. In addition, to static sink there is one mobile sink (GPS enabled), which can move across the whole WSN. Static/mobile sink has a sufficient energy resource. The sensor nodes (both super-Nodes and normal nodes) are static and have no mobility. Only mobile sink having moving capability.

Cluster heads tend to have higher capacity than regular nodes and are responsible for forwarding collected data to the sink over single or multiple hops. Both the In order to overcome the short comings observed for a static sink, the use of a mobile sink has been proposed. A mobile sink can follow different types of mobility patterns in the sensor field Such as random mobility, predictable/fixed path mobility, or controlled mobility, which has consequences with respect to energy efficiency and data collection strategies. In the following we summarize some proposed solutions for each type of mobility.

Sink mobility has higher priority for forwarding packets through the network. Note that the mobile sink may be presented at one part of the network and a cluster head which is ready to forward data is in the another part of the network, but there are other cluster heads in the interested node's radius of view .so it is possible to use the connections between the cluster heads and transmit the data to the sink from the interested cluster heads .in fact we apply the cluster heads communications to send data in situations that other cluster heads can't see the sink. 

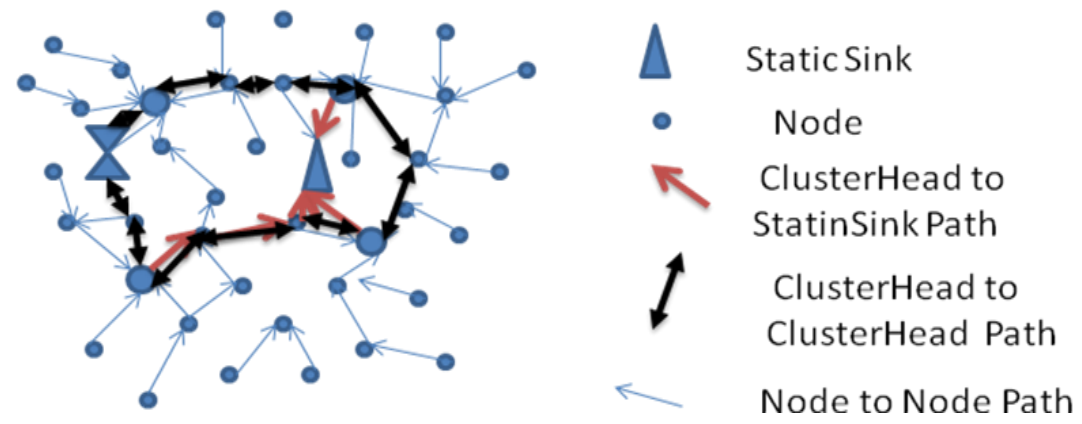

Fig. 2. This solution.

As shown in Fig. 2, the cluster Heads Only Can Connect to the Mobile Sink or Static Sink and Other Nodes Can Connect to the Cluster Heads.

The shape Of the Sink Movement is that the Mobile Sink waits for a specified time .this time is formulated by considering the history of traffic volume on the domain and the Current traffics in the domain and then Move to Next Cluster Head.

In the Other Hand it is assumed all of the Cluster Heads Have a private Path and in the presence of mobile sink in an area the connected Cluster heads will notify to other Cluster heads to send their Data to the sink by this connected $\mathrm{CH}$.

The important Note is that the Nodes Only will be able Connect to cluster heads by minimize the Connections to Static sink and Only Use the Static sink when the Mobile Sink is moving to Next Station to Connect to the Next Cluster Head.

For more Detail, In this method, the amount of energy dissipation will be decreased significantly in the nodes around the sink, as well as, since it isn't necessary that the other nodes listen to the mobile sink warning much amount of energy from the whole network energy will be decreased. Stationary sink when the mobile sink moving is adjacent to the cluster head by considering that, In time slots the MOBILE SINK is moving towards the next cluster head and because of no connection is established between the mobile sink and any cluster heads in this situation, the static sink is applied specially in real time traffics .

We should notice Must always the traffics is not uniform in all parts of the network. On the other hand video traffics are applied suddenly to the network. In this case given that we have considered a fixed move path, we can focus on the waiting time parameters.

In this work, we introduce a formula that computes the total moving time in the network according to the already observed traffic in the region. In this work we divided the moving area into the small parts of two grades and each time the mobile sink moves, the amount of traffic $(\mathrm{k})$ and transits time duration $(\mathrm{t})$ have saved. We will act according to the following formula:

$$
\text { Tnew }=\frac{\left(\left(\sum_{i=1}^{n} T i\right) * \text { Kold }\right)}{\sum_{i=0}^{n} K i}+\left(\left(\sum_{i=0}^{n} T i\right) * \frac{\left(\frac{\text { Tnew }}{\text { Knew }}-\frac{\text { Told }}{\text { Kold }}\right)}{(\mid \text { Knew }- \text { Kold } \mid)}\right)
$$

The second part of the equation is considered for the waiting time period for burst traffics, for example, multimedia traffic .in this way a sampling is performed in a little time duration. And if the amount of traffic in the similar prior waiting time duration has increased the second part value of the equation is added to the amount of waiting time.

To implement this plan, the following procedure is performed: 
A variable is considered as CANDIDATE NODE in each sensor node to avoid of creating two-way. And it is performed in the way that broadcast will be done via fixed sink. Only cluster heads have the right to receive these messages and respond to these messages. By occurring this event, all the cluster head nodes which have a parameter called StaticSinkRoute are filled with the identification of stationary sink.

These messages are broadcasted each 30 seconds. After receiving, a packet including of identification of clusters heads with the sending time is broadcasted to all networks by the cluster heads. Every fixed node that receives a packet if its CandidateNode value is empty or its deadline time has expired or the packet time is less than the current CondidateNode time; the value candidate node is replaced by current information of the receiving packet.

In this way we have clustered the network effectively without the use of heavy clustering operations based on QoS parameters.

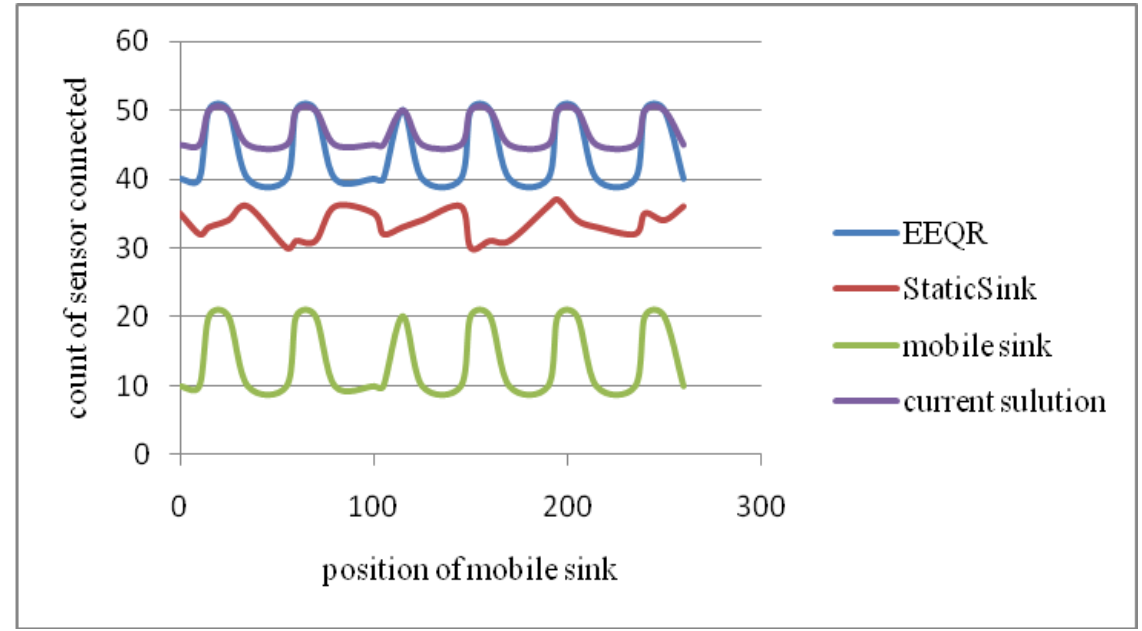

Fig. 3. Count of sensor connected to static sink in different positions.

In Fig. 3 we have compared the number of connected nodes to mobile sink in different approaches .we illustrated our proposed approach have more connected nodes to mobile sink in different positions.

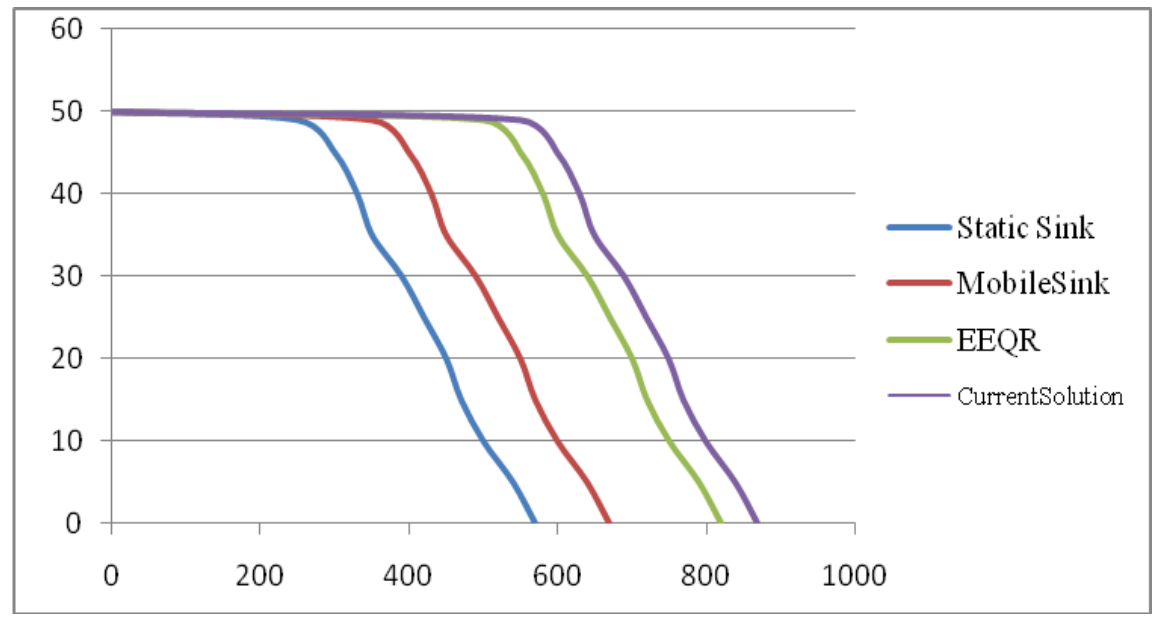

Fig. 4. Comparison of lifetime of the network in the proposed protocol and static sink and mobile sink and EEQR.

In Fig. 4 we have compared life time of Network in the proposed protocol and Static Sink and Mobile Sink and EEQR. We clearly observe the gained improvement in lifetime by this solution. 


\section{Conclusion}

In this paper we have tried to have a Stable and permanently Path to the Sink for sending Data from It by Using Mobile Sink and Static Sink and applying a Method in Type of Movement of the Mobile Sink.

In our approach, the cluster Heads Only Can connects To the Mobile Sink or Static Sink and Other Nodes Can Connect to the Cluster Heads. The shape Of the Sink Movement is that the Mobile Sink waits for a specified time this time is formulated by considering the history of traffic volume on the domain and the Current traffics in the domain and then Move to Next Cluster Head.

In the Other Hand it is assumed all of the Cluster Heads Have a private Path and in the presence of mobile sink in an area the connected Cluster heads will notify to other Cluster heads to send their Data to the sink by this connected $\mathrm{CH}$. The important Note is that the Nodes Only will be able Connect to cluster heads by minimize the Connections to Static sink and Only Use the Static sink w-hen the Mobile Sink is moving to Next Station to Connect to the Next Cluster Head.

\section{References}

[1] Thanigaivelu, K., \& Murugan, K. (2010), To alleviate congestion using hybrid sink for delay sensitive applications in wireless sensor networks. Recent Trends in Networks and Communications (pp. 431-438). Springer Berlin Heidelberg.

[2] Babar, N., \& Hasbullah, H. (2013), Energy efficient and QoS aware routing protocol for clustered wireless sensor network. Computers \& Electrical Engineering, 39(8), 2425-2441.

[3] Khan, M. I., Gansterer, W. N., \& Haring, G. (2013), Static vs. mobile sink: The influence of basic parameters on energy efficiency in wireless sensor networks. Computer Communications, 36(9), 965-978.

[4] Euisin, L., et al (2010), Communication model and protocol based on multiple static sinks for supporting mobile users in wireless sensor networks. IEEE Transactions on Consumer Electronics, 56(3), 1652-1660.

[5] Euisin, L., Park, S., Lee, J., Oh, S., \& Kim, S.-H. (2011), Novel service protocol for supporting remote and mobile users in wireless sensor networks with multiple static sinks. Wireless Networks, 17(4), 861-875.

[6] Pazzi, R. W. N., \& Boukerche, A. (2008). Mobile data collector strategy for delay-sensitive applications over wireless sensor networks. Computer Communications, 31(5), 1028-1039.

[7] Athanasios, K., \& Nikoletseas, S. (2007). Scalable data collection protocols for wireless sensor networks with multiple mobile sinks. Proceedings of IEEE 40th Annual Simulation Symposium (pp. 60-72).

[8] Yun, Y. S., \& Xia, Y. (2010). Maximizing the lifetime of wireless sensor networks with mobile sink in delay-tolerant applications. IEEE Transactions on Mobile Computing, 9(9), 1308-1318.

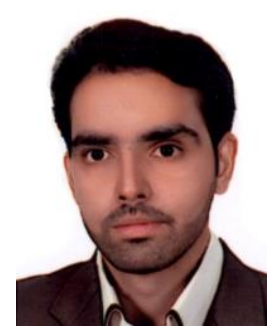

Sayyed Ahmad Hosseini was born on September 30 in 1985. He had got his B.A degree of computer engineering from Azad University of Mashhad, and M.A degree from Research and Science University of Khorasan Razavi, his personal interests in computer realm are computer hardware and computer networks.

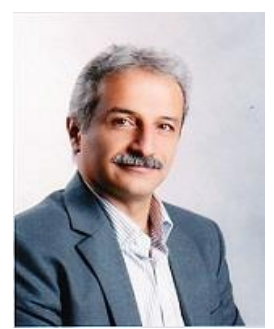

Sayyed Majid Mazinani was born in Mashhad, Iran on 28 January, 1971. He received his bachelor degree in electronics from Ferdowsi University, Mashhad, Iran in 1994 and his master degree in remote sensing and image processing from Tarbiat Modarres University, Tehran, Iran in 1997. He worked in IRIB from 1999 to 2004. He also received his $\mathrm{PhD}$ degree in wireless sensor networks from Ferdowsi University, Mashhad, Iran in 2009. He is currently an assistant professor at the Faculty of Engineering in Imam Reza 
University, Mashhad, Iran. He was the head of Department of Electrical and Computer Engineering from 2009 to 2012. His research interests include computer networks, wireless sensor networks and smart grids.

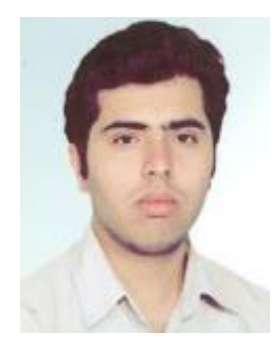

Mehdi Shahedi received the BA degree in 2011 in computer software engineering from Islamic Azad University Of Mashhad, Iran. He Received his MS degree in computer software engineering from Department of Computer, Science and Research branch, Islamic Azad University, South Khorasan, Birjand, Iran in 2014. From 2010 he has been a net and oracle developer and project manager in HRM Project In Dade Gostar Toos Co. His main research area is computer networking, including mobile sink routing protocol for delay sensitive traffic in wireless sensor network and image processing. 